\title{
Combining Social Authentication and Untrusted Clouds for Private Location Sharing
}

\author{
Andrew K. Adams ${ }^{\ddagger}$ and Adam J. Lee $\ddagger$ \\ $\dagger$ Pittsburgh Supercomputing Center \\ $\ddagger$ Department of Computer Science, University of Pittsburgh \\ akadams@psc.edu, adamlee@cs.pitt.edu
}

\begin{abstract}
Recently, many location-sharing services (LSSs) have emerged that share data collected using mobile devices. However, research has shown that many users are uncomfortable with LSS operators managing their location histories, and that the ease with which contextual data can be shared with unintended audiences can lead to regrets that sometimes outweigh the benefits of these systems. In an effort to address these issues, we have developed SLS: a secure location sharing system that combines location-limited channels, multi-channel key establishment, and untrusted cloud storage to hide user locations from LSS operators while also limiting unintended audience sharing. In addition to describing the key agreement and location-sharing protocols used by SLS, we discuss an iOS implementation of SLS that enables location sharing at tunable granularity through an intuitive policy interface on the user's mobile device.
\end{abstract}

\section{Categories and Subject Descriptors}

C.2.0 [Computer-Communication Networks]: General-Security and protection; C. 2.2 [Computer-Communication Networks] Network Protocols-Applications; K.6.5 [Management Of Computing And Information Systems]: Security and Protection-Authentication

\section{General Terms}

Security

\section{Keywords}

Key Management; Location Tracking; Presence Systems; Privacy; Security

\section{INTRODUCTION}

Over the last several years, location- and presence-sharing systems have received considerable attention from both researchers [6, $8,18,21,22,28]$ and in practice $[12,14,16,17]$. The recent explosion in mobile computing and social networking has led to deployment of a wide range of location-sharing systems (LSSs), both

Permission to make digital or hard copies of all or part of this work for personal or classroom use is granted without fee provided that copies are not made or distributed for profit or commercial advantage and that copies bear this notice and the full citation on the first page. To copy otherwise, to republish, to post on servers or to redistribute to lists, requires prior specific permission and/or a fee.

SACMAT'13, June 12-14, 2013, Amsterdam, The Netherlands.

Copyright 2013 ACM 978-1-4503-1950-8/13/06 ...\$10.00. stand-alone in nature (e.g., Google Latitude [17], FourSquare [14], or Glympse [16]) as well as integrated with other social networking platforms (e.g., Facebook places [12], Twitter, or Yelp). These types of systems allow a user to share her geographic location with her social contacts either as a first-class data object or as support for other content (e.g., attaching one's location to restaurant review). This sharing can be done in a near seamless manner, particularly when the LSS is embedded within a larger social platform.

Despite their popularity, LSSs are not without their own security and privacy problems. By their very design, these systems have the implicit shortcoming that sharing one's location with social contacts requires sharing this location with the LSS operator as well. This can lead to undesirable profiling of users by third parties, or increase users' exposure risk in the event of an LSS compromise. In addition, it is has been shown that social networks in general [29] and LSSs in particular [22] can sometimes lead to situations in which users experience regrets after (over)sharing information. This is often the result of the so-called unintended audience problem, in which data is shared with individuals other than those with whom the subject intended to share. This may manifest as a result of a location being automatically attached to content posted on a social network, accidental sharing of a location with a user's entire set of contacts instead of a restricted subset, or posting a location that contradicts other statements made by the user [22].

The latter problem is symptomatic of both LSS and access control complexity. For instance, it is well-known that users social networks have many more contacts than they interact with on a dayto-day basis: a 2011 poll of 1,954 British citizens found that the average person had 476 Facebook friends, but only 152 contacts in their cellular phone [31]. Furthermore, research studies have shown that users frequently make mistakes when authoring even basic access control policies in commodity systems [4, 11,23]. As such, it is clear that accidents and misconfigurations can lead to oversharing in large social networks. On the other hand, the problem of required sharing with LSS operators is one of economic incentives: the ability to study user habits and carry out targeted advertising provides revenue for operators of the systems.

An interesting observation, however, is that current generation smartphones are capable of helping mitigate both of the above types of concerns. Given the $3 \mathrm{G} / 4 \mathrm{G}$ connectivity of these devices and the open APIs to cloud storage-as-a-service (SaaS) providers like Amazon S3 [1], it is possible for mobile applications to explicitly manage a user's published location history. Furthermore, smartphones store rich information about a users' close contacts (e.g., email addresses, phone numbers) and have access to multiple channels of communication (e.g., WiFi, 3G/4G, Bluetooth, SMS). As a result, it is possible to develop robust key exchange protocols-e.g., based on multiple distinct avenues of communication and histori- 
cal context, or by leveraging location-limited channels-that allow location data to be selectively encrypted prior to upload, thereby preventing snooping attack by the SaaS provider and limiting incidences of over-sharing.

In this paper, we describe Secure Location Sharing (SLS), a decentralized LSS that leverages the above observations to limit the over-exposure of user location data without relying on trusted infrastructure. Specifically, SLS allows users to set up secure location sharing with selected contacts by pairing devices in one of two ways. Users who happen to be physically co-located can use location-limited visual channels to pair devices (similar to [20]). Users who are located apart from one another can instead leverage multiple communication channels (e.g., email and SMS) along with contextual question/answer protocols to help prevent man-inthe-middle (MitM) attacks during device pairing. Keys established during this pairing process are then used to aid in securely sharing a user's location at a tunable granularity (e.g., GPS coordinate, citylevel, etc.) via untrusted SaaS services. In exploring SLS, we make the following contributions:

1. We demonstrate the first decentralized LSS that is capable of providing flexible and secure location sharing over untrusted infrastructure. Unlike existing approaches to securing social networks (e.g., X-pire! [2]) our work does not involve abuse of existing social network APIs, but rather builds secure and flexible sharing into the real-life social networks managed by users' smartphones.

2. We propose an alternate economic model for LSSs, in which the users providing their location to others pay for the storage used to host their data. ${ }^{1}$ This removes the economic incentives driving traditional LSS providers to view user location histories, and further reduces the risk of accidental overexposure due to LSS compromise.

3. SLS limits the unintended audience sharing problem by requiring explicit device pairing between providers and consumers of location sharing. By leveraging multi-channel and/or location-limited pairing protocols, this setup procedure is robust against even very strong adversaries with control over large portions of the network environment.

4. We develop an iOS application as a proof-of-concept implementation of SLS. This demonstrates both the efficiency of our approach, as well as the simplicity of interfaces needed to manage the secure device pairing aspects of SLS.

The rest of this paper is organized as follows. In Section 2, we discuss related work, and briefly explain the problems associated with canonical location-sharing or presence system. We discuss our goals, properties and principals of our system in Section 3. In Section 4 we present our framework and implementation for secure location sharing. Section 5 re-examines our design, evaluates the performance and security of the system, and explores our directions for future work. We present our conclusions in Section 6.

\section{RELATED WORK}

Google Latitude [17], FourSquare [14], Facebook Places [12], and Glympse [16] are examples of LSSs that operate by having users upload their location data to the service, such that others (i.e.,

\footnotetext{
${ }^{1}$ Note, however, that some SaaS providers provide lower-tier service that is sufficient for SLS at little to no cost to the user (e.g., http: //aws.amazon.com/free/).
}

consumers) can access the location data. Current strategies for addressing privacy issues in LSSs are typically based on obfuscating the location data or anonymizing the provider; the efficiency of these techniques are discussed in, e.g., [26,27]. In [24], the authors address oversharing in LSSs by providing users with interactive feedback about the number of users accessing their location, and the frequency of these accesses. Our work deviates from prior work by (i) preventing the LSS from viewing a user's location data, and (ii) by ensuring that a user has full control over who she chooses to share her location data with and how she intends for her location to be consumed.

The protection and secrecy of a user's data contained in the cloud is the focus of DataLocker [9], which is a collection of tools that enable a provider to encrypt data prior to uploading the data to the cloud. Our model does this precisely with location data, however, our model is not tied to any specific cloud entity. Moreover, we do not generically encrypt location data: policy dictates the precision with which data is presented to the consumers, and how it is protected in the cloud. Instead of protecting data, X-Pire! [2] attempts to decay data (ostensibly images, but the technique could be applied to location data) by associating a key to an image; when the key expires the X-pire! aware server refuses to serve the data. Similarly, Vanish [15] decays data by altering links to the data stored within a DHT. Although our model does not address the decaying of data, it could benefit from techniques like these in the future.

Several papers present advanced key management protocols that make use of smart phone technology [7, 13, 19, 20]. McCune et al. describe the protocol, Seeing-is-Believing (SiB) [20], in which the camera in users' smart phones capture 2D barcodes-these 2D barcodes are used as commitments for exchanging public keys. Our key management protocol relies heavily on the concepts and ideas introduced presented in this work. SafeSlinger [13] is a protocol and framework designed to exchange public keys between smart phones; this is precisely one of the tasks that our key management protocol is designed to accomplish; our work diverges from [13] by (i) using the location-limited channel between pairing smart phones to fully exchange asymmetric keys, and (ii) by leveraging what we refer to as a file-store deposit (a pointer to a dropbox) to assist in symmetric key management. Accelerometer data from two smart phones is used in [19] and [7] to aid in authentication for secure pairing. Mayrhofer et al. [19] employ a strategy of shaking two phone simultaneously to generate a movement limited channel, while BUMP [7] uses the accelerometer and location data between to bumping smart phones. Again, our work differs from pairing protocols based on movement limited channels, by operating over location-limited and multichannel communication channels.

Multichannel security protocols, as surveyed in [30], are ways to mitigate against MitM attacks by using multiple communication channels, e.g., radio, visual and 1-bit on/off or toggle buttons, during authentication. The idea is that a malicious eavesdropper cannot eavesdrop on all channels. We use an instantiation of this idea in the variant of our pairing protocol based on historical, multiple open-lines of communication.

Limitations of Prior Work. As alluded to in Section 1 and Section 2, LSS have significant privacy issues, and in fact the primary issue was exposed in [5]. In this study, it was shown that that users are uncomfortable with a service controlling access to their location data. Techniques have been introduced to mitigate users' privacy issue concerns, e.g., data can be diffused, or aggregated, but all of these reduce the utility of the data. This is especially troubling if the providers' intentions are for their data to be consumed at a high precision by a specific user, or one or more groups 


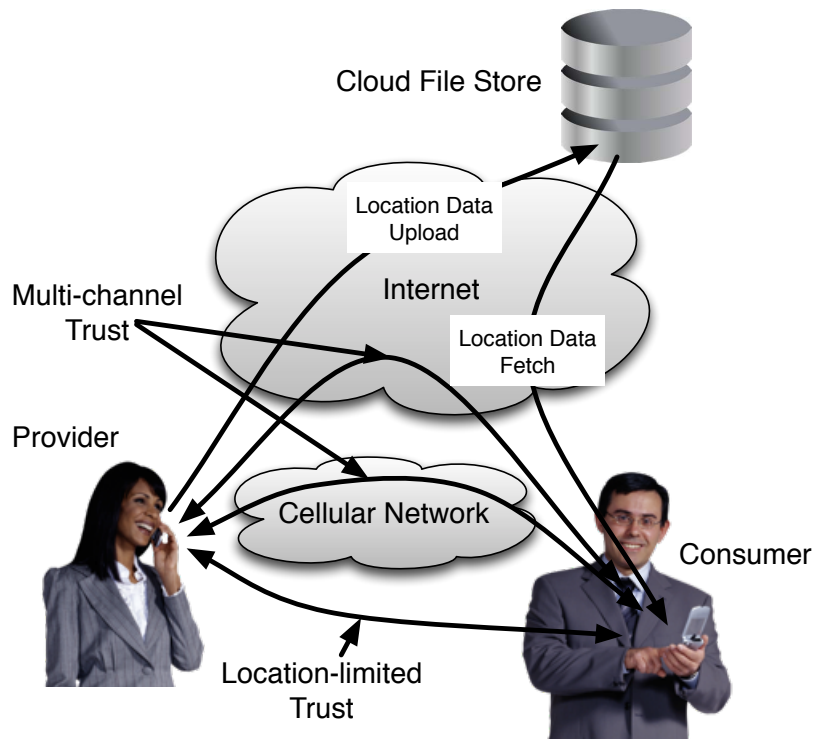

Figure 1: SLS Architecture Overview.

of users. A secondary issue that arose in the study is that many users are uncomfortable knowing that anyone can see their location information, i.e., once the location data is uploaded to the LSS the user forfeits control over the data.

Although not novel, the combination of symmetric and asymmetric cryptography can help address both of these concerns: i.e., providers encrypt their location data prior to uploading it to a LSS, and then must distribute the decryption $\mathrm{key}(\mathrm{s})$ to enable retrieval by authorized consumers. However, this key management process can be a heavy burden. McCune et al. and Farb et al. [13,20] observe that smart phones are fast becoming ubiquitous and are exceptionally portable and, as such, are usually available during vis-a-vis interactions. This makes smartphones an ideal platform for bootstrapping the exchange of cryptographic keys through the locationlimited channels that can exist between two parties. We further observe that current smart phones possess the technology to perform key management efficiently and fully over location-limited channels, but only lack the protocols and framework to achieve this. SafeSlinger [13] is architected to rely on Internet connectivity to/from a server to aid in the key exchange protocol (i.e., SafeSlinger only uses the location-limited channel between the pairing smart phones for initializing the key exchange, and then for confirmation). This has the obvious disadvantages of requiring (i) that the server be available at all times, and (ii) that the exchange occurs in an environment that possesses network connectivity. We argue that both of these requirements are unnecessary to exchange asymmetric keys in a close, vis-a-vis setting that leverages location-limited channels, while using current smart phone technology.

\section{SYSTEM DESIGN}

The SLS system was designed with two main goals in mind: (i) enabling tunable and private location sharing with limited contacts, and (ii) limiting end-user location over exposure. We now overview our system architecture and describe the threat model within which we expect SLS to be used.
Architecture Overview. Figure 1 presents a high-level view of the SLS system. Users in the system can be divided into two classes: providers and consumers. Providers share their location with others, while consumers retrieve the locations of others; a user can act as both a provider and a consumer. We assume that all users have smartphones, as well as (perhaps self-signed) asymmetric key pairs. Providers' smartphones must be able to detect their current location, e.g., via GPS or cellular/WiFi localization. Secure location sharing is enabled by shared, symmetric keys. The sharing of these symmetric keys is facilitated by asymmetric keys exchanged during a device pairing protocol. SLS provides two pairing protocols to exchange asymmetric keys: one based upon in-person communication over location-limited channels, and another that leverages multi-channel communication for situations in which in-person exchange is not possible. To pair devices using location-limited channels, users' smartphones must have the ability to read and decode QR codes. To pair devices using multiple, historical open-lines of communication, users' smartphones must have both network access (e.g., via WiFi), as well as the ability to send and receive SMS messages. Although the multi-channel based pairing protocol requires that principals have previously communicated, there is no such restriction within the location-limited pairing protocol. Encrypted location data is shared through the use of a SaaS service (e.g., Amazon S3) contracted by the provider. Note, although one can currently find SaaS services that are free, our model assumes an associated cost to use the service. We require that the SaaS service allow any user to download data posted to the provider's account. We do not require all providers to use the same SaaS service.

Adversary Model. In this paper, we make the following assumptions. We first assume that user smartphones are free of malware, as this would immediately make user locations available to the adversary through the smartphone API. We assume that all network communications are subject to read, replay, reorder, and modification by a Dolev-Yao style adversary [10]. Finally, we assume that the SaaS providers used are honest-but-curious in nature. That is, we assume that they will correctly execute the GET and PUT operations provided by their APIs, but may try to derive provider locations by inspecting the data that they host. In this work, we do not address DoS/DDoS attacks against SaaS providers as a means of thwarting location sharing.

\section{SECURE LOCATION SHARING}

We now describe the design of the Secure Location Sharing (SLS) framework. We first describe how multiple granularities of location data are encrypted and managed by the provider (Section 4.1). Then, we describe two protocols for pairing provider and consumer devices to enable secure retrieval of provider locations from SaaS services (Sections 4.2-4.3). Next, we discuss the policy controls available to providers within SLS (Section 4.4). Finally, we describe our iOS implementation of SLS (Section 4.5).

\subsection{Location Sharing}

In SLS, a provider's smartphones is responsible for capturing her location data using, e.g., WiFi/cellular localization or GPS. Each location sample collected by SLS is represented as a four-tuple containing a location coordinate, an estimate of the provider's speed of travel, the providers bearing/heading, and a timestamp indicating when the sample was collected. In total, each location sample collected by SLS requires approximately 200 bytes to store. Given that a provider may wish to share her location at multiple granularities, the sample collected by SLS is generalized to each desired 
granularity or precision prior to upload (e.g., exact, neighborhood, county, or state precision). These (perhaps generalized) provider locations are shared with consumers via an (untrusted) SaaS service contracted by the provider. As such, location data must be cryptographically protected prior to upload. To accomplish this, the provider generates one symmetric key for each granularity level at which her data is to be shared, and then CBC encrypts each sample prior to upload.

Encrypted location samples are thus unreadable to the SaaS service, with whom the user is under no obligation to share her location (unlike in a traditional LSS). We note, however, that there is economic incentive for the SaaS service to correctly house the data, regardless what the data's contents are: users are not bound to a particular SaaS service and can simply migrate their data should the SaaS service misbehave. Further, providers also have complete control over the amount of information shared: they may post only a single "current" location (e.g., by overwriting a single location sample), or instead maintain a history of location samples (e.g., by storing a sliding window of $n$ location samples). In SLS, we refer to these two operational modes as update and history, respectively. Finally, consumers are under no obligation to create accounts with an LSS, as all data is pulled from SaaS providers by SLS using HTTP GET requests made to world-readable URLs.

Of course, the reliance of SLS on symmetric keys to protect provider location data raises two issues. First, it must be possible for providers and consumers to securely authenticate one another and exchange the cryptographic material needed to retrieve location data at the desired level of granularity. To this end, we present protocols for device pairing based on location-limited and multichannel protocols in Sections 4.2 and 4.3, respectively. Second, it must be both possible and efficient for the provider to alter the list of consumers with whom she shares information and the granularity at which this information is shared, which are challenges that are addressed in Sections 4.2 and 4.4.

\subsection{Location-Limited Pairing}

In-person interactions are an ideal setting for device pairing and key exchange. These interactions present the users engaging in the pairing protocol with an opportunity to physically identify the owner of the device with which they are attempting to establish a secure channel, as well as enable the use of location limited (e.g., visual [20]) channels to exchange data. The combination of humanto-human authentication and device-to-device communication that is difficult to intercept results in demonstrative identification [3] of the participants in location-limited device pairing protocols. SLS utilizes the traits of visual, location-limited channels, and extends the concepts presented in $\mathrm{SiB}$ [20] when pairing devices to aid in symmetric key management.

Figure 2 illustrates our location-limited device pairing protocol, which we call Communionable Trust $(C T)$. This protocol makes use of human-to-human audio and visual communication, as well as device-to-device visual communication using on-board cameras and Quick Response (QR) codes. The first step of this protocol is the real-world identification and authentication of the humans who wish to pair devices to facilitate location sharing via SLS. After the human participants have agreed to pair devices, the remainder of the protocol focuses on the exchange of public key information between provider and consumer, and exchanging metadata that enables the sharing of both symmetric keys and location data.

In Step 2 of the protocol, the consumer generates a QR code that contains her public key $\left(K_{C}\right)$ as well as a device identity token $\left(I D_{C}\right)$ used to associate her device with her real-world identity, as managed by the provider's smartphone. Figure 3 shows a QR code

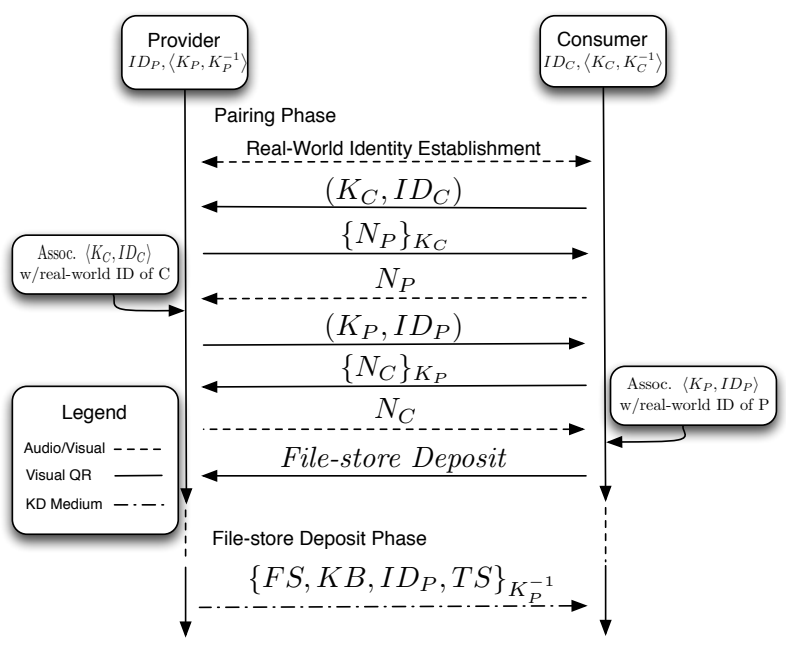

Figure 2: Communionable Trust Protocol.

containing a 1024-bit RSA public key and it's associated identity token. ${ }^{2}$ The provider scans this code with his phone, and recovers $K_{C}$ and $I D_{C}$. He then generates a random challenge nonce, $N_{P}$, encrypts $N_{P}$ using $K_{C}$, and generates a QR code containing the resulting ciphertext (Step 3). The consumer scans this QR code, decrypts the resulting ciphertext, and verbally communicates the nonce value to the provider (Step 4). After verifying this exchange, the provider associates $K_{C}$ and $I D_{C}$ with the consumer's contact information in his smartphone.

This process is then mirrored in Steps 5, 6, and 7 of the communionable trust protocol, which provides the consumer with the providers public key $\left(K_{P}\right)$ and identity token $\left(I D_{P}\right)$.

In the final step of the Pairing Phase, the consumer QR-encodes their file-store deposit-a description of an out-of-band channel over which the consumer wishes to be notified of the provider's SaaS store-and presents it for the provider to scan. This message is sent unencrypted due to the location-limited nature of the visual channel used by this protocol. The use of this consumerspecified "drop box" allows the provider to inform the consumer asynchronously (e.g., via SMS) if they change SaaS providers at a later date, and thus obviates the need to re-execute the CT protocol.

File-store Deposit Phase. The final message of the communionable trust protocol handles the distribution of metadata that enables the consumer to retrieve location samples uploaded by the provider. This message is sent over the channel identified in Step 8 of the communionable trust protocol, and is a four-tuple of values containing a URI for the file store at which the provider's location data will be hosted $(F S)$, a URI at which the consumer can access her shared key bundle $(K B)$, the provider's identity token $\left(I D_{P}\right)$, and a timestamp $(T S)$. The entire message is then signed by the provider to ensure authenticity. After using $T S$ and $K_{P}$ (which is associated with $I D_{P}$ by the consumer) to validate the freshness and authenticity of this message, $F S$ and $K B$ provide the consumer with all of the information that is needed to securely access the provider's location data.

\footnotetext{
${ }^{2}$ We note that version 40 QR codes can encode approximately 1500 bytes of data, which is more than sufficient for exchanging even 2048-bit public keys.
} 


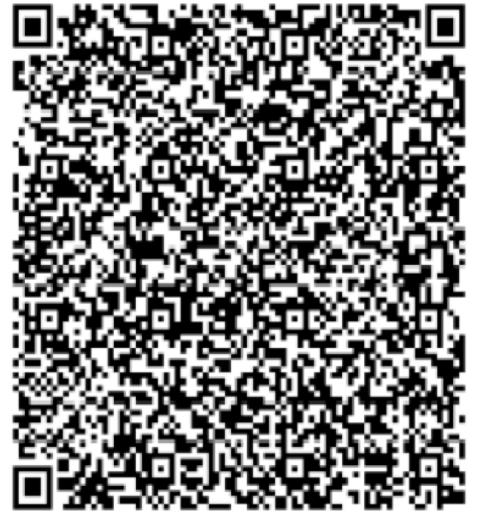

Figure 3: QR-encoded 1024-bit RSA public key (with associated identity token).

The key bundle URI, $K B$, provides the consumer with a pointer to an encrypted key bundle stored on the provider's SaaS service. This key bundle is a (key, version, signature) three-tuple that is encrypted using the consumer's public key $\left(K_{C}\right)$. The key field of this tuple contains the current symmetric key corresponding to the precision level with which the consumer is permitted to access the provider's location, the version field indicates the version of this key, and the signature field is a hash of the key and version fields encrypted with provider's private key $\left(K_{P}^{-1}\right)$. Key versions are used to facilitate location retrieval as keys change in response to changes in provider access controls (see Section 4.4). The level of indirection added by the key bundle-as opposed to directly transferring keys as part of the CT protocol-eliminates the need for direct communication between the provider and consumer upon every policy change. After recovering their key bundle, the consumer can easily retrieve provider locations from the file store URI, $F S$, and decrypt this data.

\subsection{Multi-channel Pairing}

It is unreasonable to assume that users of SLS will always have the ability to physically co-locate during the device pairing process. As such, we also describe a pairing protocol that can be used by individuals who are not within close proximity. As such protocols can be vulnerable to MitM attacks, we make use of multiple historical, open-lines of communication associated with principals on their smart phones (e.g., email address, phone number, or instant messaging account). In this context, historical refers to pre-existing contacts, and open-lines of communication implies that the principals have communicated with the preexisting contact over those multiple channels. This combination of properties gives providers (resp. consumers) higher assurance that the identity of the consumer (resp. provider) is correct, since (i) existing contact information is used to bootstrap the communication process and (ii) an active attacker would need to control multiple communication channels to subvert the protocol. Our Historical Communication Channels (HCC) protocol is described in Figure 4.

HCC is initiated in the first step of the Pairing Phase by the consumer, who sends their public key $\left(K_{C}\right)$ and device identity token $\left(I D_{C}\right)$ used to associate her device with her real-world identity

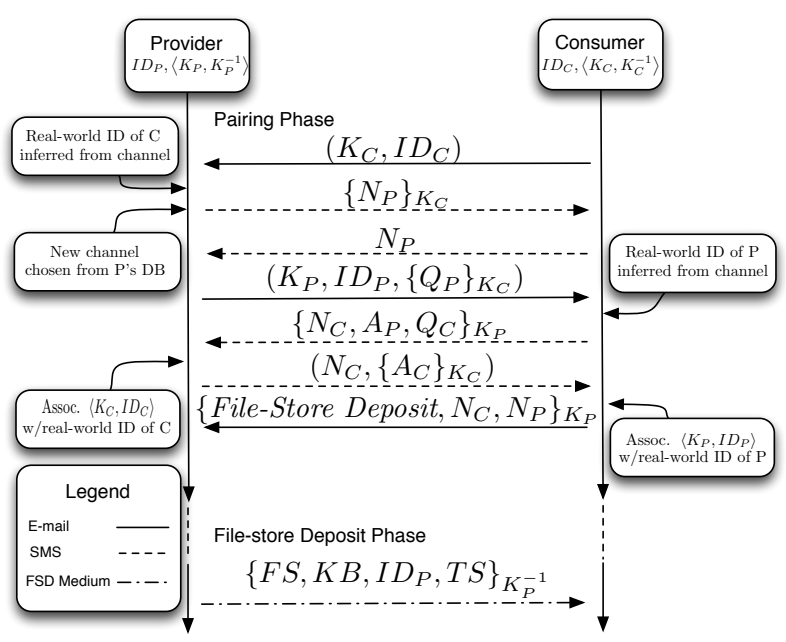

Figure 4: Historical Communication Channels Protocol.

(which is established through previous contact as managed by the provider's smartphone). This message is sent to the provider over an existing communication channel (e.g., a known email address), signified using a solid line in Figure 4. Upon receiving $K_{C}$ and $I D_{C}$, the provider generates a random challenge nonce $N_{P}$, encrypts $N_{P}$ using $K_{C}$, and sends $\left\{N_{P}\right\}_{K_{C}}$ to the consumer via a different historical, open-line of communication that the provider has previously associated with the consumer (e.g., via SMS), which is denoted by a dashed line in Figure 4 . The consumer decrypts the ciphertext and returns $N_{P}$ back to the provider over $\mathrm{HCC}$ Secondary (Step 3). At this point in the protocol, the provider is confident that the consumer has access to the private key associated with the public key received in Step 1.

However, the provider does not yet have a high level of confidence that the consumer is indeed whom the provider believes they are (e.g., someone other than the consumer could have stolen the consumer's smart phone). Hence, the provider generates a $s e-$ cret question $\left(Q_{P}\right)$ that, within reason, only she and the consumer should know the answer to; e.g., "Who was the away team at the last hockey game that we attended together?". $Q_{P}$ is encrypted with $K_{C}$ and is sent to the consumer along with $K_{P}$ and $I D_{P}$ over the primary channel (Step 4). The consumer generates (i) the answer $\left(A_{P}\right)$ to $Q_{P}$, (ii) her own random nonce $N_{C}$, and (iii) her own secret question $\left(Q_{C}\right)$. All three are encrypted with $K_{P}$ and sent to the provider via HCC Secondary (Step 5). If, after decrypting the resulting ciphertext, $A_{P}$ is correct the provider sends $N_{C}$ and her answer $\left(A_{C}\right)$ encrypted with $K_{C}$ via HCC Secondary (Step 6). After verifying $A_{C}$, the consumer encrypts their file-store deposit (File-Store Deposit), $N_{P}$ and $N_{C}$ with $K_{P}$ and sends them via HCC Primary (Step 7).

Finally, similar to the CT protocol, the provider assigns the consumer's precision level and the File-store Deposit Phase begins (see Section 4.2 and 4.4). We note that the HCC protocol should be terminated if either (i) a principal receives a secret question via SLS without first initiating or receiving a public key from the same principal over a different channel, or (ii) the response to a participant's secret question is incorrect.

\subsection{Policy Control}

As alluded to in the Section 4.2, after learning the consumer's 
public key and file-store deposit (either through CT or HCC), the provider must associate the consumer with the precision level at which they are authorized to view the provider's data. All consumers that are assigned the same precision level by a provider are considered to be in the same group and, thus, all have access to a single symmetric key protecting location disclosures made at this precision level. As a result, the symmetric key associated with a particular precision level may need to be updated as the group of users who have access to that precision level changes over time.

To provide the highest level of security for the provider's location data-i.e., preserving forward- and backward-secrecy-these shared symmetric keys should be changed whenever a consumer is added to a precision group or removed from a precision group. The former case ensures that new consumers cannot access old data, while the latter ensures that former consumers cannot access new data. Altering the symmetric key for a particular precision level requires creating a new symmetric key, encrypting key bundles for each user authorized at this precision level, and depositing the bundles on the provider's file store. After asynchronously retrieving these new key bundles, authorized consumers can again access the providers data. While shared symmetric key update is non-trivial, our evaluation (Section 5.1) shows that the overheads associated with this process in practice are minimal. We note that it is not necessary for the provider to re-pair their device with consumers via CT or HCC, as the asymmetric keys use for key management are not affected by a consumer's change in precision level.

We recognize that our LSS model prevents the enforcement of certain policies found in existing LSSs; e.g., policies that enable location sharing only when two parties are within a certain physical proximity, or policies that place access count limits on individual users. LSSs that can implement proximity-based policies are able to do so because they have access to the location data of all their users, and can thus determine the distance between two users. Since our goal is to prevent the LSS from acquiring this information, this type of policy can not easily be enforced in SLS. Enforcing constraints on access frequency is also enabled via LSS intervention, which is contrary to our assumed sharing model. We do note, however, that the ability to enforce these types of policies would be a worthwhile addition to SLS. However, we defer the exploration and development of techniques for achieving these goals to future work.

\subsection{Implementation}

SLS was implemented as an iOS iPhone application. It was installed on an iPhone $4 \mathrm{~s}$, and the location-sharing and CT pairing protocol were evaluated via the IPhone $4 \mathrm{~s}$ and an iPhone simulator (modified to behave as if it could scan the iPhone 4s' public keys).

Precision Levels. Our SLS implementation collects and stores provider locations as GPS coordinates, and provides four precision levels at which a these locations can be shared. The precision levels supported are exact, neighborhood, state, and none. Support for the neighborhood and state sharing levels is provided by masking lower-order bits in the exact GPS coordinates stored within SLS.

Management Interface. The utility and usability of a security system's policy interface is crucial to its successful use: the ability to clearly indicate who can access an individual's data at what precision is key. We approached this in SLS by presenting the provider with a clean, simple display that consists of a list of principals (i.e., smartphone-managed identities associated with each consumer), and the precision-level at which each consumers have been authorized (see Figure 5). The precision level can easily be changed

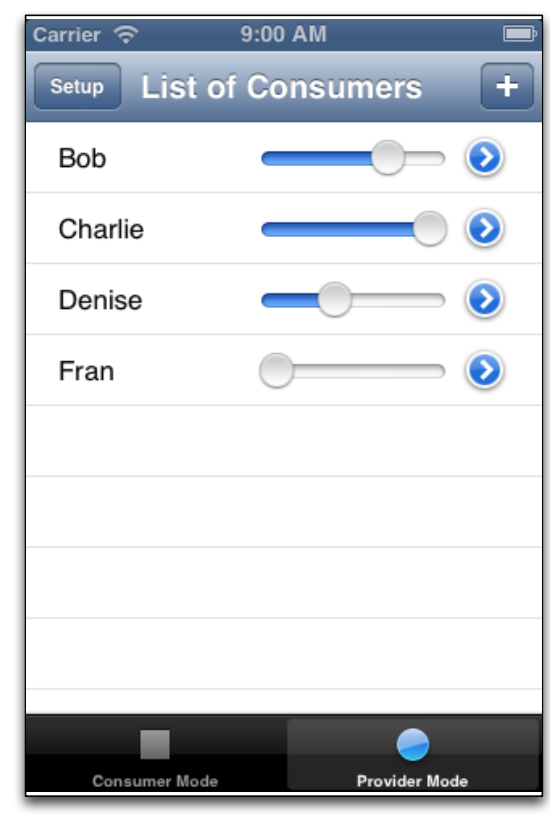

Figure 5: List of Alice's Consumers in SLS.

by the provider by adjusting a slider within this interface. Additionally, a detail view icon at the end of each row allows the provider to immediately review the consumer's information, resend the consumer's shared key bundle URL, or delete the consumer.

New consumers can easily be added when the provider taps the $[+]$ button in the upper-right corner of the List of Consumers view (see Figure 5). This presents the provider (or the consumer, when they navigate into their respective Add Provider screen) with a choice of either the location-limited, CT-based key pairing protocol, or the HCC pairing protocol to exchange public keys and the consumer's file-store deposit.

Figure 6 shows several steps of the CT device pairing process, as executed by the provider and consumer. In the first step, the provider (Alice) has entered the consumer's identity (Bob), and Bob has done the same for Alice. When both tap the QR-code button, SLS will present both the provider and consumer with the task list associated with device pairing in CT (Step 2). The provider and consumer will iterate through the steps, synchronizing when $\mathrm{QR}$ images are printed and scanned, or during challenge/responsesthese steps have been omitted from the Figure for clarity. In Step 7, the provider taps the button to scan the consumer's file-store deposit as a QR code, and Step 8 shows the consumer's screen after displaying their file-store deposit QR code to the provider.

SaaS Support. Our current implementation of SLS supports the use of Amazon's S3 services as a cloud file-store. ${ }^{3}$ The period at which SLS updates GPS coordinates and then uploads the location data is configurable by the provider. ${ }^{4}$ For simplicity, our imple-

\footnotetext{
${ }^{3}$ An example file-store URL using Amazon's S3 service is: https://s3.amazonaws.com/id-precision-hash/ locationdata.b64, where id-precision-hash is a hash of the provider's identity token and the precision level assigned to this location data.

${ }^{4} \mathrm{iOS}$ has two settings for location gathering, the first operates using a distance filter to determine when a new location update should occur, the second is a power-saving mode, in which a location update
} 

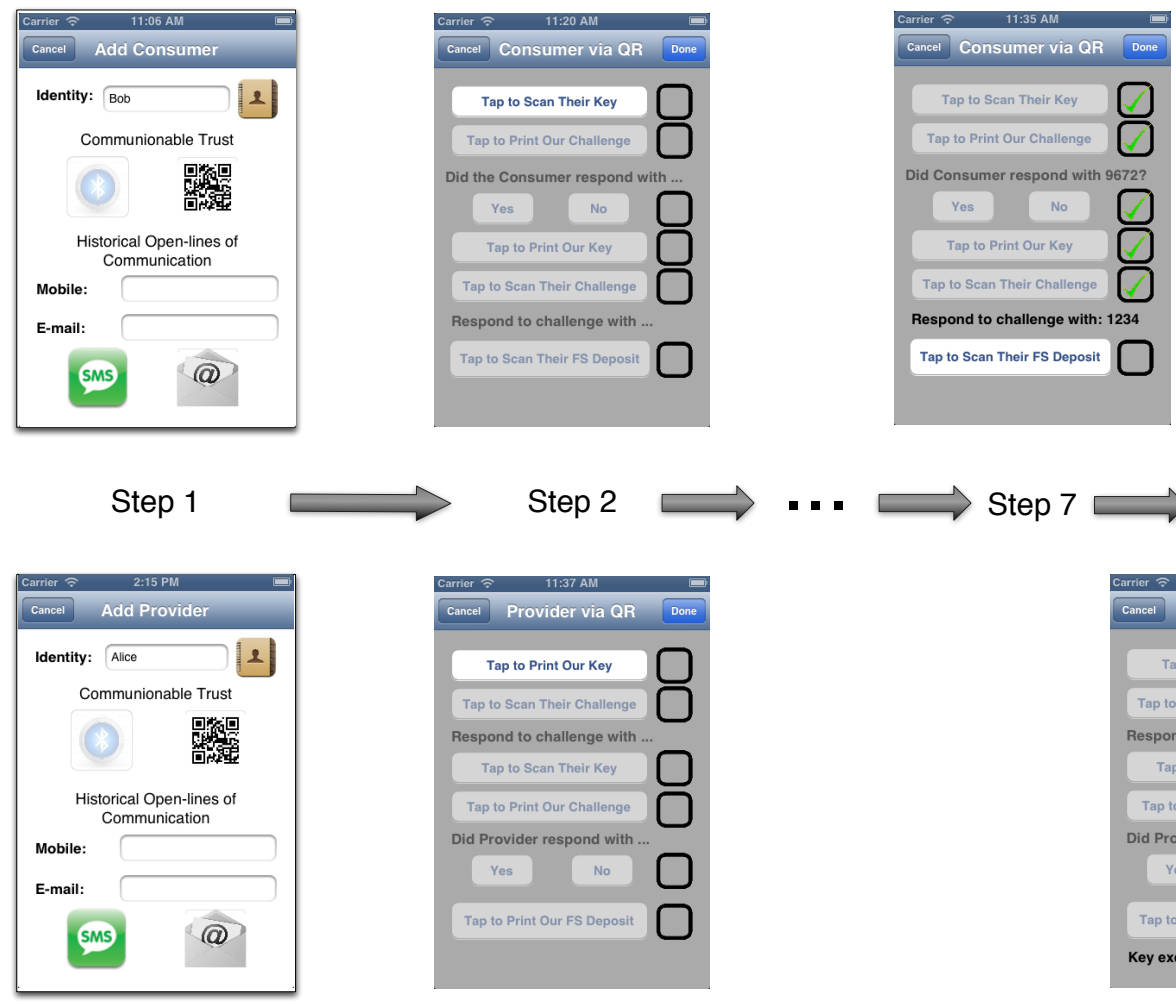

Step 2

Step 7

Step 8
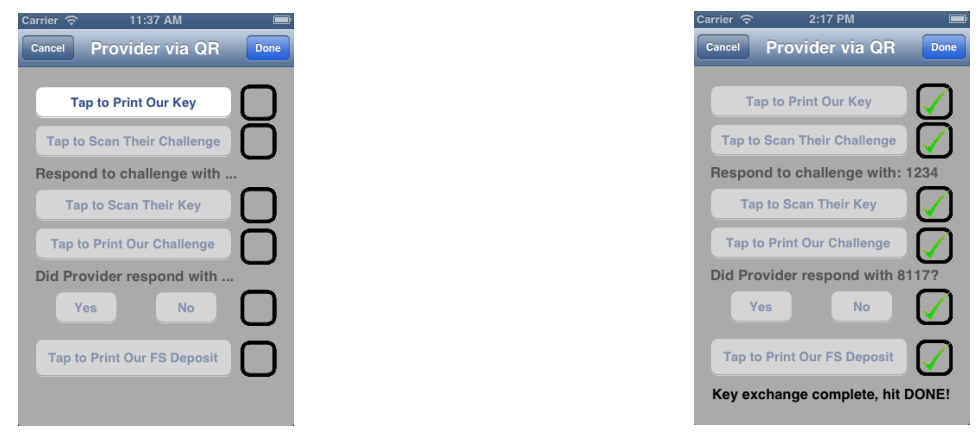

Figure 6: Screenshots of Communionable Trust protocol implemented in SLS. Steps 1, 2 and 7 are shown for the provider, and Steps 1,2 , and 8 are shown for the consumer.

mentation used the iOS Core Library API to serialize the location data that was gathered for the provider, with the resulting serialized location objects being $1 K B$ in size. Although these objects are larger than those that could be produced by a custom serializer, the encryption and transmission overheads associated with these larger objects are not significant on the iPhone $4 \mathrm{~s}$.

Amazon S3 is also used to store encrypted key bundles for the consumers associated with a given producer. Whenever a consumer is added or a consumer's precision level is changed, the shared keys (for all precision-levels involved) are regenerated, new key bundles are generated and uploaded to the provider's S3 store, and all future location samples are encrypted using the new key. Upon detecting a key version mismatch, the SLS application used by a consumer automatically fetches the new key bundle from S3. The new key can then be used to decrypt more recent location updates, which may be stored in either update or history mode. To support history mode, updates are stored in a log file referred to as the history $\log$, which contains an entry for each location data update in the window. The entries consist of the location data, time stamp and a signature over a hash of the two components. Thus, a consumer can check the history log to fetch any updates they may have missed, as their periodicity can be set differently than the providers.

Map View. The consumer's initial view displays their providers' positions on a map (see Figure 7). If the provider is operating in history mode, in which a log of location data updates is being kept in their file-store, the consumer can view the provider's location as a path. Since the location data stored on S3 also contains the bear- only occurs during a "significant" location change. Both modes are supported in our implementation.

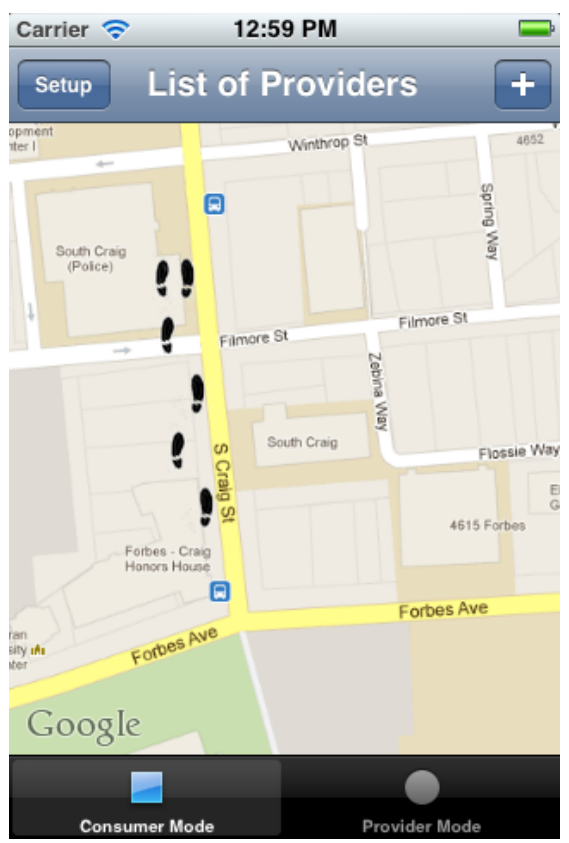

Figure 7: Consumer's view of a provider walking up the street. 
ing of the provider (in addition to the location coordinate), SLS can plot the provider's location using the entire history that it can obtain. This resulting view is a trail of footprints corresponding to the provider's path along the map. Tapping on a provider's footprints displays the provider's name and a details disclosure button, which leads to that provider's detailed information view.

\section{DISCUSSION}

We developed a system for enhancing user privacy during location sharing by adopting and extending previous work in device pairing, and tapping into the ubiquity and availability of cloud services. Our system was architected to be both scalable and secure, while also preserving providers' sharing policies and affording utility to consumers. We now discuss both the performance of our SLS implementation, and assurance provided by our pairing protocols.

\subsection{Performance Evaluation}

In evaluating the runtime overheads of SLS, we break our analysis into four phases: the device pairing phase, the file-store deposit phase, location data upload by providers, and location data download by consumers. To evaluate the communionable trust protocol (Section 4.2), we carried out 15 pairings using our iOS implementation of SLS and found the average time required for this process was 110 seconds. Although this pairing process takes longer than, e.g., Bluetooth device pairing, the overheads are reasonable given the human effort required by this protocol (i.e., scanning $\mathrm{QR}$ codes). We did not evaluate the time required by the historical communication channels protocol (Section 4.3) as this protocol was designed to be run asynchronously over multiple higher latency channels (e.g., email and SMS).

After devices are paired using either the CT or HCC protocol, the provider sends the consumer a digitally signed message that includes URIs that are to be used to retrieve the consumer's key bundle and the provider's location data. This data can only be sent after the provider has indicated the precision level with which the consumer may access his information, and is returned to the consumer over a communication channel identified in the final message of the pairing phase of the CT or HCC protocol (i.e., the file-store deposit message). The overhead associated with this phase is linear in the number of consumers, but is only a one time cost, as this only occurs once per consumer (or in the wake of the rare event of the provider changing cloud services). In our iOS implementation of SLS, the generation of the signature on this message took $98 \mathrm{~ms}$ on average over 15 runs

A consumer that is tracking $n$ providers must retrieve $n$ encrypted location samples from up to $n$ different SaaS providers. The frequency with which this process occurs is a parameter that can be set within our implementation by the consumer. We measured the average time required to execute the HTTP GET command required to obtain a provider's encrypted location sample and execute the symmetric key decryption of this sample. When using Amazon S3 as SaaS provider, this process took approximately $40 m s$ per provider.

On the other hand, the cost incurred by the provider during uploads is constant: an encrypted location sample must be uploaded at each of the $p$ precision levels used by the provider's policy. The provider can choose the periodicity that new GPS coordinates are produced, so the constant time cost can be applied once per day, or several times per minute (depending on how fast the smart phone is moving and how fast it can generate new GPS coordinates). In our iOS implementation, encrypting and uploading location samples at three precision levels over 15 runs took on average, $3.7 \mathrm{~s}$, with a standard deviation of $1.8 \mathrm{~s}$.

\subsection{Security Analysis}

The SLS system was designed with two main goals in mind: (i) enabling tunable and private location sharing with limited contacts, and (ii) limiting end-user location over exposure. This is achieved by storing encrypted location samples on SaaS servers contracted by the provider, and leveraging location-limited or multichannel pairing protocols to facilitate the key management required by this approach. We now informally analyze the security afforded by the protocols developed in this paper.

Location-limited Pairing Protocol. The communionable trust protocol described in Section 4.2 provides principals with high assurance regarding the secure handling of location data. In particular, the face-to-face nature of this protocol allows the human device owners to authenticate each other in the most natural sense. As a result, the public keys exchanged and validated using this protocol are intrinsically tied to the real-world participants in the protocol, since anyone attempting to launch a MitM attack would be quite conspicuous - we refer the reader to the security analysis in [20] for a thorough examination of attacks against this type of channel, as well as comparisons against other channels (e.g., audio, infrared, physical contact, etc.). For this reason, the principal's public key and identity exchanged via CT are made available to other applications outside of SLS. ${ }^{5}$ Assuming that the consumer keeps her private key a secret, the public key obtained during this process enables the provider to safely transmit symmetric keys needed to recover his location to the consumer without exposing his location to unauthorized individuals (including the SaaS provider).

Multi-Channel Pairing Protocol. In settings where the provider and consumer are not physically located together, obtaining the assurance level of the communionable trust protocol is difficult. The protocol in Section 4.3 attempts to overcome the lack of physical proximity in three ways. First, it leverages historical communication channels managed by each user's smartphone to increase assurance in the identity of the party being communicated with, thereby reducing the likelihood of accidental sharing with inappropriate parties. Second, the HCC protocol makes use of multiple communication channels to decrease the likelihood of a successful MitM attack against the protocol. In examining Figure 4, we can see that an adversary with access to only the e-mail channel has the ability to inject public keys into the protocol, but cannot complete the validation process for these keys. Likewise, an adversary with access to only the SMS channel can cause parties in the protocol to reject valid public keys, but cannot inject their own public keys.

While this protocol cannot protect against a MitM attack when the adversary has access to both channels used by the protocol, as long as the implementation ensures that both channels are indeed distinct (e.g., WiFi + SMS, as opposed to $3 \mathrm{G} / 4 \mathrm{G}+\mathrm{SMS}$ ) the cost to mount such an attack would be prohibitive to most. A more realistic attack vector against cell phones would be a physical attack, i.e., the attacker steals the smartphone of the consumer (resp. provider). However, the third protection mechanism in HCC does allow it to protect against this attack. Specifically, each party is required to answer a contextual "secret question" (i.e., $Q_{P}$ and $Q_{C}$ in Figure 4) proposed by the other party prior to finalizing the pairing process and enabling location sharing. Unless the individual possessing the stolen smartphone has intimate knowledge of the relationship between the provider and consumer, they would be unable to answer this type of question and, thus, the protocol would fail.

\footnotetext{
${ }^{5} \mathrm{iOS}$ provides for this by using a shared or public group within the key-chain.
} 
Although this question-and-answer mechanism is useful, it is not perfect. Moreover, although the protocol requires that the implementation leverage distinct communication channels, the mechanisms used to ensure the channels are distinct could themselves be attacked, i.e., an attacker could try to trick the implementation to use multiple channels that he controls. For these reason, we encourage principals to upgrade their public keys exchanged via HCC with CT, whenever the opportunity presents itself.

Shared Key Management. The shared or symmetric key management scheme employed by SLS provides three assurances to its users, including; the assurance that only a provider can upload new key material to the cloud file-store, the assurance that the location data can not be accessed by anyone without the appropriate shared key, and the assurance that exposure of the identities of the provider's consumers is mitigated within the cloud file-store. The first assurance is ensured by the act of encrypting the shared key bundle with the consumer's public key, while including a version and signature, i.e., an adversary can not alter the contents of the bundle as it is signed. The adversary could replay (or overwrite) an older shared key bundle, however, as the version of the key is included in the bundle the consumer will know that the current shared key bundle is incorrect.

Since the shared key bundles are encrypted with the consumers' public keys, and the uploaded location data is encrypted with the shared keys contained within those bundles, the provider is assured that only those consumers possessing the appropriate shared key will be able to view the provider's location data. Granted, a consumer could make the symmetric key accessible to non-authorized users, however, we note that this is the bane of all shared key security systems, and its mitigation is outside the scope of this paper.

Finally, exposure of the consumers' identities is mitigated through the use of the file-store deposit. Specifically, the provider can use any unique token to identify a consumer's shared key bundle, as the location of the shared key bundle is delivered to the consumer out-of-band via the file-store deposit. Admittedly, the cloud service will know how many consumer a provider has, and possibly how many precision levels are in use by the provider (by examining the contents of the history log, if in use). Thus, in order for the cloud service to uniquely identify a consumer, they'll have to rely on properties of the HTTP connection during the fetch (e.g., IP address, HTTP message headers).

\subsection{Beyond Location Sharing}

Interestingly, although our system was designed to share location data, there is no reason that the protocols and framework constituting SLS could not be adopted for other types of data (e.g., documents, pictures, music, or videos). That being said, we do not envision these SLS-like services replacing forums like Flickr or YouTube, which have proven to be de-facto file stores for widely sharing information. However, these SLS-like services could be useful for providing secure hosting for information that is to be shared with a more limited audience.

\subsection{Future Work}

The most pertinent area of future work involves conducting a user study to assess the utility and ease-of-use of our SLS implementation. Although this paper focused on the correctness and feasibility of a system like SLS, ensuring that the system is indeed usable is also quite important. A user study of our prototype iOS implementation could help answer questions about the usability of the policy interface built into the application, as well as about the ability of users to manage their privacy using SLS. Insights from exit surveys conducted with participants in such a study could also help guide the design of more intuitive sharing interfaces, and protections that might help further limit unintended audience sharing (e.g., short-term sharing settings, etc.).

Another area that we intend to pursue is the development of more advanced and cooperative policy controls.For example, consider a provider that only feels comfortable sharing her location data with consumers in the same region. We believe that this could be accomplished, for example, if the pair had mutual sharing configured between them (i.e., both acted as providers and consumers), and secure function evaluation was leveraged to decide when the distance between the two principals crossed some threshold (at which point SLS would operate normally). Although such advanced controls are likely possible, making these controls both intuitive for the user and efficient for the device to execute could prove to be challenging in practice.

Another intriguing extension of SLS's key management protocols would be implementing support for a type of key escrow for emergency response. Specifically, the symmetric key used in encrypting high-precision location data could not only be encrypted with the consumers' public keys, but also broken into shares, to be distributed to emergency respondents using a threshold scheme (e.g., [25]). For example, consider a 2 -of- $n$ threshold scheme in which key shares are distributed to the local police force for the provider's municipality, the state police, and the security contractor used by the provider's employer. In this case, for instance, the provider's employer could cooperate with the local authorities in the event that the provider was missing long enough for the employer to file a missing persons report. Further developing these sorts of policy-based extensions to SLS could prove to be an interesting area of study.

\section{CONCLUSIONS}

location-sharing systems (LSSs) have high utility, but recent research has shown that (i) many users are wary of sharing detailed trace information with LSS operators and (ii) the large social networks with which LSSs are often integrated make it all too easy to accidentally share location data with unintended audiences. In this paper, we make inroads to the above problems by developing SLS, a framework for private location sharing that combines the use of social authentication protocols based upon location-limited or multi-channel protocols, with user-contracted cloud SaaS providers to facilitate secure data storage and location sharing. In particular, our device pairing protocols leverage the smaller social networks managed by user smartphones to provide a high degree of assurance in the identities of the individuals with which sharing is to occur. The asymmetric keys exchanged during this process can then be used to distributed shared symmetric keys that protect a location provider's sensitive location information from unauthorized viewers, including the cloud service used to host the data. Our iOS prototype implementation of SLS shows that the overheads associated with this form of key management are reasonable.

Although the information sharing model developed in this paper was developed to facilitate secure location sharing based upon an individual's real-world social contacts, we believe that our techniques have application beyond location sharing. In particular, the combination of device pairing with high identity assurance and third-party storage that is used by SLS can be used to facilitate the sharing of many types of information currently shared using existing social networks (e.g., photos, etc.) without requiring implicit trust in the operators of these social networks. 
Acknowledgements. This research was supported in part by the National Science Foundation under awards CNS-0964295 and CNS-1017229.

\section{REFERENCES}

[1] Amazon s3. https://s3.amazonaws.com/.

[2] J. Backes, M. Backes, M. Dürmuth, S. Gerling, and S. Lorenz. X-pire! - a digital expiration date for images in social networks. CoRR, abs/1112.2649, 2011.

[3] D. Balfanz, D. K. Smetters, P. Stewart, and H. C. Wong. Talking To Strangers : Authentication in Ad-Hoc Wireless Networks. In ISOC Network and Distributed Systems Security Symposium (NDSS), 2002.

[4] L. Bauer, L. F. Cranor, R. W. Reeder, M. K. Reiter, and K. Vaniea. Real life challenges in access-control management. In CHI 2009: Conference on Human Factors in Computing Systems, pages 899-908, April 2009.

[5] J. T. Biehl, E. Rieffel, and A. J. Lee. When privacy and utility are in harmony: Towards better design of presence technologies. Personal and Ubiquitous Computing, 2012.

[6] J. T. Biehl, E. G. Rieffel, and A. J. Lee. When privacy and utility are in harmony: towards better design of presence technologies. Personal and Ubiquitous Computing, 17(3):503-518, 2013.

[7] Bump. http://bu.mp.

[8] Y. Cai and T. Xu. Design, analysis, and implementation of a large-scale real-time location-based information sharing system. Proceeding of the 6th international conference on Mobile systems applications and services MobiSys 08, page 106, 2008.

[9] Datalocker. http: //www appsense.com/labs/data-locker.

[10] D. Dolev and A. C. Yao. On the security of public key protocols. In Proceedings of the 22nd Annual Symposium on Foundations of Computer Science, SFCS '81, pages 350-357, Washington, DC, USA, 1981. IEEE Computer Society.

[11] S. Egelman, A. Oates, and S. Krishnamurthi. Oops, i did it again: mitigating repeated access control errors on facebook. In CHI 2011: Conference on Human Factors in Computing Systems, pages 2295-2304, 2011.

[12] Facebook places. http://www. facebook.com/ about/location?_fb_noscript $=1$.

[13] M. Farb, M. Burman, G. Singh, C. Jon, and M. A. Perrig. Safeslinger: An easy-to-use and secure approach for human trust establishment.

http: / / www. cmu.edu/homepage/computing/ 2012/winter/safeslinger.shtml.

[14] Foursquare. https://foursquare.com/.

[15] R. Geambasu, T. Kohno, A. Levy, and H. M. Levy. Vanish: Increasing data privacy with self-destructing data. In Proc. of the 18th USENIX Security Symposium, 2009.

[16] Glympse. http: / /www.glympse.com/.

[17] Google latitude.

https://www.google.com/latitude/.

[18] J. Lindqvist, J. Cranshaw, J. Wiese, J. Hong, and
J. Zimmerman. I âĂŹ $m$ the mayor of my house : Examining why people use foursquare - a social-driven location sharing application. Design, 54(6):2409-2418, 2011.

[19] R. Mayrhofer and H. Gellersen. Shake Well Before Use: Intuitive and Secure Pairing of Mobile Devices. IEEE Transactions on Mobile Computing, 8(6):792-806, June 2009.

[20] J. McCune, A. Perrig, and M. Reiter. Seeing-Is-Believing: Using Camera Phones for Human-Verifiable Authentication. In IEEE Symposium on Security and Privacy, pages 110-124. IEEE, 2005.

[21] B. Palanisamy and L. Liu. Mobimix: Protecting location privacy with mix-zones over road networks. Data Engineering, International Conference on, 0:494-505, 2011.

[22] S. Patil, G. Norcie, A. Kapadia, and A. J. Lee. Reasons, rewards, regrets: Privacy considerations in location sharing as an interactive practice. In Symposium on Usable Privacy and Security (SOUPS), July 2012.

[23] R. W. Reeder, L. Bauer, L. F. Cranor, M. K. Reiter, K. Bacon, K. How, and H. Strong. Expandable grids for visualizing and authoring computer security policies. In $\mathrm{CHI}$ 2008: Conference on Human Factors in Computing Systems, pages 1473-1482, 2008.

[24] R. Schlegel, A. Kapadia, and A. J. Lee. Eyeing your exposure: quantifying and controlling information sharing for improved privacy. In Proceedings of the Seventh Symposium on Usable Privacy and Security, SOUPS '11, pages 14:1-14:14, New York, NY, USA, 2011. ACM.

[25] A. Shamir. How to share a secret. Commun. ACM, 22(11):612-613, 1979.

[26] R. Shokri, G. Theodorakopoulos, G. Danezis, J.-P. Hubaux, and J.-Y. Le Boudec. Quantifying Location Privacy: The Case of Sporadic Location Exposure. In The 11th Privacy Enhancing Technologies Symposium (PETS), 2011.

[27] R. Shokri, G. Theodorakopoulos, J.-Y. Le Boudec, and J.-P. Hubaux. Quantifying Location Privacy. In 2011 Ieee Symposium On Security And Privacy (Sp 2011), IEEE Symposium on Security and Privacy, pages 247-262. Ieee Computer Soc Press, Customer Service Center, Po Box 3014, 10662 Los Vaqueros Circle, Los Alamitos, Ca 90720-1264 Usa, 2011.

[28] J. Y. Tsai, P. Kelley, P. Drielsma, L. F. Cranor, J. Hong, and N. Sadeh. Who's viewed you?: the impact of feedback in a mobile location-sharing application. In Proceedings of the SIGCHI Conference on Human Factors in Computing Systems, CHI '09, pages 2003-2012, New York, NY, USA, 2009. ACM.

[29] Y. Wang, S. Komanduri, P. Leon, G. Norcie, A. Acquisti, and L. Cranor. I regretted the minute i pressed share: A qualitative study of regrets on facebook. In Symposium on Usable Privacy and Security (SOUPS), 2011.

[30] F. L. Wong and F. Stajano. Related Work in Multichannel Security Protocols. IEEE Pervasive Computing, 6(4):31-39, 2007.

[31] www.MyVoucherCodes.co.uk. Average brit has 476 facebook friends compared to 152 mobile phone contacts, 2011. 